\title{
Photonic Provisioning Using a Packaged SOI Hybrid All-Optical Wavelength Converter in a Meshed Optical Network Testbed
}

\author{
Christos Stamatiadis, Dimitrios Kalavrouziotis, Annachiara Pagano, Roberto Morro, Emilio Riccardi, \\ Leontios Stampoulidis, Karsten Voigt, Giovan B. Preve, Ludwig Moerl, Jochen Kreissl, Kennedy Landles, \\ Stephen Duffy, Hercules Avramopoulos, Lars Zimmermann, and Klaus Petermann
}

\begin{abstract}
We demonstrate path wavelength routing and switching using a packaged and pigtailed hybrid integrated silicon-on-insulator (SOI) photonic circuit. The fabricated device incorporates a $1.25 \mathrm{~mm}$ nonlinear semiconductor optical amplifier mounted on the SOI board and two multimode interference-based cascaded delay interferometers monolithically integrated on the same SOI substrate. The photonic chip has a footprint of 384 $\mathbf{m m}^{2}$ and performs all-optical wavelength conversion at ultrafast data rates. The packaged and pigtailed module is inserted and tested as a switching node in a meshed optical network testbed carrying real data traffic and encompassing a wavelength division multiplexing optical link. Optical path wavelength switching and routing is implemented by a generalized multiprotocol label switching control plane. We present $10 \mathrm{GbE}$ video-streaming routing as well as $40 \mathrm{~Gb} / \mathrm{s}$ wavelength switching employing the system integrated all-optical wavelength converter. Error-free transmission and different kind of end-to-end services (video distribution, audio conference, and video conference) have been successfully evaluated.
\end{abstract}

Index Terms-All-optical signal processing, all-optical wavelength conversion, all-optical wavelength routing, hybrid photonic integration, silicon-on-insulator (SOI).

Manuscript received April 19, 2012; revised July 08, 2012; accepted July 13, 2012. Date of publication July 17, 2012; date of current version August 24, 2012. This work was supported by the European Commission through project ICT-BOOM (FP7-224375) under the 7th Framework Programme.

C. Stamatiadis, K. Voigt, and K. Petermann are with the Joint Lab Silicon Photonics HFT4, Technische Universitaet Berlin, 10587 Berlin, Germany (e-mail: christos.stamatiadis@tu-berlin.de; karsten.voigt@tu-berlin.de; petermann@tu-berlin.de).

D. Kalavrouziotis and H. Avramopoulos are with the Photonics Communications Research Laboratory, National Technical University of Athens, 15773 Athens, Greece (e-mail: dkalav@mail.ntua.gr; hav@mail.ntua.gr).

A. Pagano, R. Morro, and E. Riccardi are with Transport Innovation, Telecom Italia Lab, 10148 Turin, Italy (e-mail: annachiara.pagano@telecomitalia.it; roberto.morro@telecomitalia.it; emilio.riccardi@telecomitalia.it).

L. Stampoulidis is with Constelex Technology Enablers, Corallia Microelectronics Innovation Center, 15125 Athens, Greece (e-mail: 1s@constelex.eu).

G. B. Preve is with Nanophotonics Technology Center, Universidad Politecnica de Valencia, 46022 Valencia, Spain (e-mail: gpreve@ntc.upv.es).

L. Moerl and J. Kreissl are with Fraunhofer-Institut für Nachrichtentechnik, Heinrich-Hertz-Institut, 10587 Berlin, Germany (e-mail: ludwig.moerl@hhi.fraunhofer.de; jochen.kreissl@hhi.fraunhofer.de).

K. Landles and S. Duffy are with Optocap Ltd., Livingston, EH54 7DQ, U.K. (e-mail: Kennedy.Landles@optocap.com; Stephen.Duffy@optocap.com).

L. Zimmermann is with the Joint Lab Silicon Photonics HFT4, Technische Universitaet Berlin, 10587 Berlin, Germany, and also with IHP Microelectronics, Leibniz-Institut, 15236 Frankfurt, Germany (e-mail: lars.zimmermann@tu-berlin.de).

Color versions of one or more of the figures in this paper are available online at http://ieeexplore.ieee.org.

Digital Object Identifier 10.1109/JLT.2012.2209172

\section{INTRODUCTION}

$\mathbf{G}$ LOBAL IP traffic is currently experiencing a huge data explosion [1], compelling network operators to adopt alternative architectures, resize existing infrastructures, and apply faster solutions [2]. As bandwidth demands surpass the capacity of electronic interfaces [3], power consumption jumps over the eco-friendly limitations [4], hardware expenses outstrip the budget of telecom operators [5], and routing topologies continue to follow complex and parallelization approaches [6], signal processing becomes a real pressure to service providers that are staggering for miniaturized, ultrahigh performance integrated devices. To alleviate temporally the data overload, they have turned their focus on minimizing capital and operational expenses by simplifying routing topologies, sharing resources, and collapsing routers through virtualization architectures [7]. But as the annual traffic growth increases, even such topologies are no longer applicable, making essential the use and exploitation of different concepts, materials, and components.

With the recent advances in functional photonic integration, ultracompact, scalable, and high-capacity superchips have emerged outperforming the capabilities of electronic equipment [8]. The technology strategy employed so far is based either on InP [9] or silicon [10], with both platforms being in controversy for dominating and hosting the future integrated circuits. Blending however the properties of each substrate, photonic components can become more powerful, compact, and efficient, turning optical networking into a stable and promising approach [11]. But the material incompatibility has been a barrier for years, raising issues on hybridization procedures, bonding quality, component alignment, and uniformity. Recently, fabrication techniques have become more flexible, precise, and mature, allowing the placement of InP active devices on silicon interfaces with good mechanical, electrical, and optical performance. The integration techniques applied so far vary from heterogeneous processes [12] to hybrid integration approaches [13], [14] with different operational characteristics, performance metrics, and employing different kind of waveguides (nanowires or intermediate silicon waveguides).

Thick silicon-on-insulator (SOI) $(\sim 4 \mu \mathrm{m})$ exhibits some grateful properties allowing low polarization dependence, low insertion loss, close match to the spot-size of III-V devices, and fabrication capabilities in any silicon foundry. Based on these attributes, a series of high-speed all-optical wavelength 


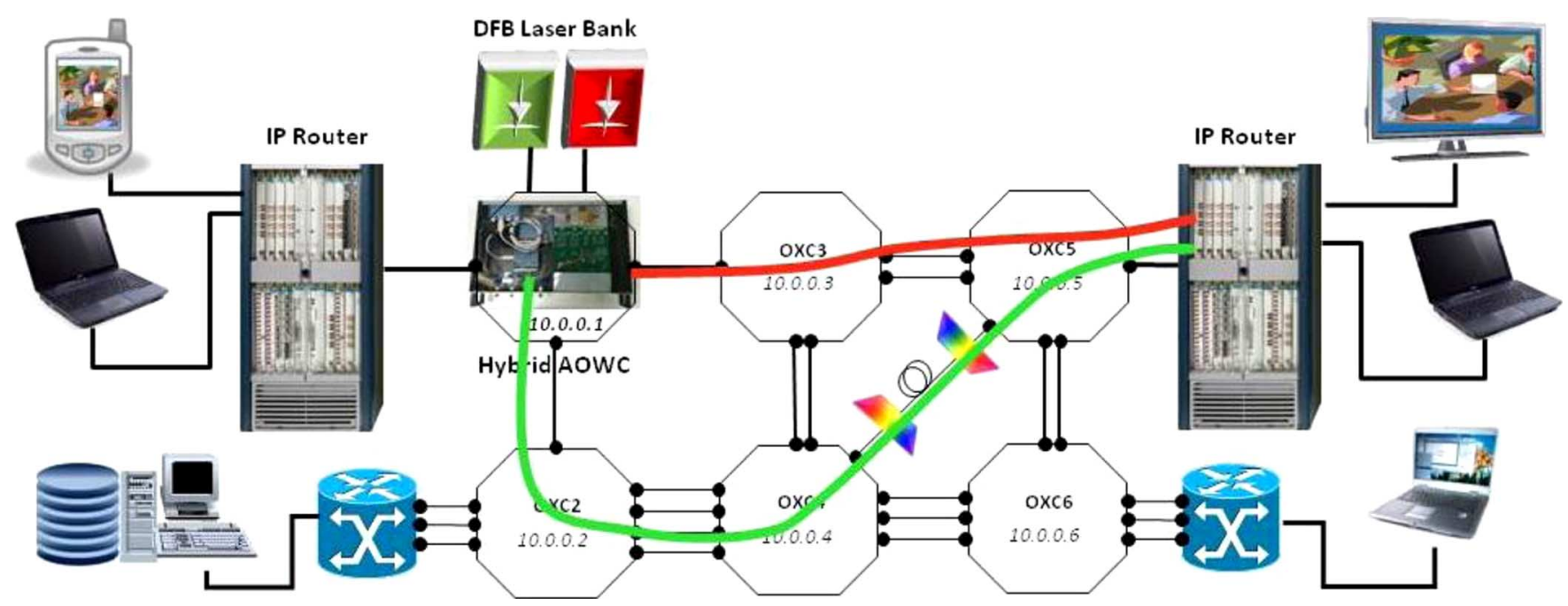

Fig. 1. Routing concept.

converters (AOWC) has been currently integrated and system tested up to $160 \mathrm{~Gb} / \mathrm{s}$ employing the Au-Sn sputtering and flip-chip soldering technique [15]. All these optical components however have been evaluated as standalone devices showing their operational potential in application-specific functionalities without revealing their actual performance in network system environments. And the main reason for this is that the photonic elements are provided unpackaged and with no pigtails, making their insertion and interconnection to system traffic interfaces impractical. But as all-optical wavelength conversion gains momentum in broadband photonic routing architectures [16], it is the time for truly compact and packaged devices to show their proof-of-principle performance in network demonstrations.

Following this rationale, we present in this paper path video streaming routing and data switching at 10 and $40 \mathrm{~Gb} / \mathrm{s}$ employing a packaged and pigtailed high-speed AOWC in a meshed optical network testbed. The photonic chip incorporates a $1.25 \mathrm{~mm}$ nonlinear semiconductor optical amplifier (SOA) mounted on the SOI board using gold-tin bumps as small as $14 \mu \mathrm{m}$. The device performs chirp filtering and signal polarity inversion with two multimode interference-based cascaded delay interferometers (DIs) monolithically integrated on the same SOI substrate. The system packaged module is inserted in a real network carrying real data traffic, encompassing a wavelength division multiplexing (WDM) link and triggered by a generalized multiprotocol label switching (GMPLS) control plane. We demonstrate quality of service $(\mathrm{QoS})$ measurements and error-free transmission performance for different routing scenarios using a truly integrated photonic switching element.

\section{ROUTING CONCEPT}

Fig. 1 depicts the proof-of-principle concept for transmitting and switching real data traffic through the hybrid AOWC. Two IP routers with $10 \mathrm{GbE}$ optical interfaces have been interconnected as client signals and routed across a meshed optical network. The optical network comprises a hybrid integrated SOI AOWC, a distributed feedback (DFB) laser bank, multiple switching nodes, and a dense wavelength division multiplexing (DWDM) link. The AOWC comprises a hybrid integrated
SOA for cross gain and phase modulation (XGM-XPM) and two concatenated DIs for chirp filtering and signal polarity inversion [17]. The photonic circuit is packaged, pigtailed, and system integrated together with customized electronic driving boards in a rack mountable case. Wavelength selection for the final wavelength-based routing is performed with an optical switch controlled by a GMPLS control plane. According to the wavelength seeded in the AOWC, data traffic is routed either directly to the final client (red line) or to a DWDM link (green line) and subsequently to the end user. The testbed employed is a GMPLS meshed network with fiber switching capabilities made around with a commercial optical switching matrix (3-D MEMS) logically partitioned to emulate five nodes (OXC2 to OXC6) interconnected by bundles of bidirectional fibers. A further emulated node (OXC1) implements the switching function via the hybrid AOWC device followed by a DWDM arrayed waveguide grating (AWG) demux to address the output ports. Every node has its own controller (Linux-based PC) running a GMPLS control plane stack, interconnected with the controllers of the neighbor nodes by means of point-to-point Fast Ethernet control channels. Some of the nodes have ports used to interconnect client devices (e.g., IP routers) seen as UNI interfaces, both at data and control plane level. Signalling and routing protocols are RSVP-TE and OSPF-TE, respectively, with GMPLS extensions. In one of the links, a commercial 100 GHz spacing DWDM system with $125 \mathrm{~km} \mathrm{G.652} \mathrm{fiber} \mathrm{spools}$ is inserted, being part of a transport regional/metropolitan network with $40 C$-band channels, optical add-drop multiplexing nodes, optical amplifiers, and dispersion compensating modules. QoS verification is implemented with packet loss and performance quality end-to-end measurements.

To explore the switching capabilities of the hybrid AOWC at high-speed data rates, we generate $40 \mathrm{~Gb} / \mathrm{s}$ bit streams and we assess wavelength conversion process and path routing performance through bit-error-rate (BER) measurements. Data traffic is transmitted and routed either to OXC3-OXC5 or to OXC2-OXC4-OXC6-OXC5 according to the pump wavelength used for driving the hybrid AOWC. Video data transmission and path switching at $40 \mathrm{~Gb} / \mathrm{s}$ was not feasible 

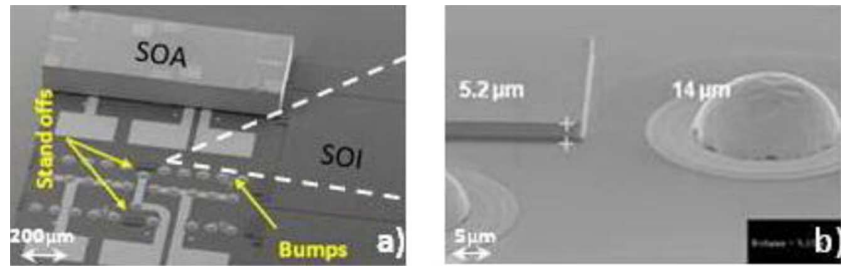

Fig. 2. (a) SOI board stand-offs and Au-Sn bumps. (b) Hybrid integrated AOWC.

since $40 \mathrm{GbE}$ IP router optical interfaces were not available in our network setup.

\section{HYBRID AOWC FABRICATION}

The hybrid integration technology employed for the fabrication of the high-speed AOWC is based on the Au-Sn flip-chip soldering technique. The solder material is deposited by sputtering followed by a lift-off process, while the hybrid process facilitates optical and electrical integration between the SOI motherboard and the SOA. Adaptation of the standard fabrication technology is required both on the silicon as well as on the III-V side. The external electrical interface (dc) is implemented on the SOI motherboard, which is wired to the SOA landing area by an $\mathrm{Au}$ interconnect. The flip-chip adaptable SOA is designed with a buried heterostructure with p-n current blocking layers incorporating laterally tapered input/output waveguides serving as spot-size converters. The SOA has a length of $1.25 \mathrm{~mm}$ and exhibits saturation power of $10 \mathrm{dBm}$, noise figure of $7 \mathrm{~dB}$, internal single pass gain of $22 \mathrm{~dB}$ (maximum value $25 \mathrm{~dB}$ ), and polarization sensitivity lower than $1.5 \mathrm{~dB}$. For proper horizontal and vertical optical alignment, alignment marks and dry etched trenches serve as counterparts to the board stand-offs. The interplay of these counterpart areas with the stand-offs of the SOI board determines the vertical alignment, which is performed passively, whereas horizontal alignment is executed actively with a fine-placer via alignment marks.

For the SOI board fabrication, three processing steps are required: SOI waveguide etch and integration zone processing by etching through buried oxide and etching of alignment stands, complete metallization of SOI motherboard with electrical interconnects, and under bump metallization (UBM) and $\mathrm{Au}-\mathrm{Sn}$ bump sputtering. Fig. 2(b) illustrates a close SEM image of stand-offs and $\mathrm{Au}-\mathrm{Sn}$ bumps with appropriate height for SOA flip-chip mounting. Optical integration is achieved by waveguide butt-coupling, posing a major challenge in terms of alignment. The spot sizes involved are in the range of $2-3 \mu \mathrm{m}$, which necessitates a flip-chip alignment precision well within a $1 \mu \mathrm{m}$ window. This precision cannot be achieved by standard pickand-place equipment, but requires use of high-precision flipchip tools. The flip-chip mounting of the SOA into the SOI substrate is being performed by a high accuracy flip-chip bonder (FC-150 with $1 \mu \mathrm{m}$ alignment precision) that takes over the positioning against the stand-offs and the thermal cycling for bump reflow and soldering. The reproducibility/uniformity of bump ball fabrication and the lift off the required postprocessing (optimized reflow and soldering) is achieved after a series of trial tests resulting to devices with proper mechanical stability and contact quality. The AOWC screening process involved manual

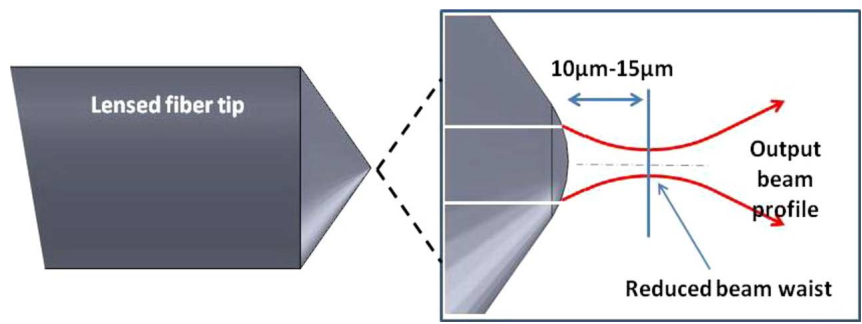

Fig. 3. Schematic of conical lensed fiber tip.

shear tests, microscopic inspection of the SOI motherboards, SEM inspection of the AOWC boards, and fiber optical measurements on the flip-chip assembled devices. Fig. 2(a) depicts the fabricated hybrid AOWC comprising the flip-chip bonded SOA and the two concatenated DIs.

\section{HYBrid AOWC PACKAGING}

The packaging of the hybrid AOWC introduced a number of challenges. First, the physical size $(32 \mathrm{~mm} \times 12 \mathrm{~mm})$ would require the use of a highly planar mounting surface to avoid wafer warping during attach. Warping has been known to introduce degradation in optical performance during device operation. Additionally, no back-side metallization was present which forced the use of epoxy/adhesive attach processes. Moreover, for aligning an array of fibers, the common method would be to use a single v-groove array component (FVA) and pre-epoxy attach each fiber with subsequent end-face polishing. This method however was not employed for two reasons. First, the SOI device had a $4 \mathrm{~mm}$ pitch which is much larger than standard offthe-shelf FVAs. Second, lensing would be required to provide best optical coupling performance through mode-matching the fiber output to the mode dimension of both the SOA and output waveguides.

It is common practice in high reliability telecom packages to introduce lensing as part of a fiber end-face preparation and remove the need for additional bulk optics within the package. Typically, the fiber end face is first prepared with a rotationally symmetric conical taper, with subsequent rounding of the fiber tip which generates an equivalent geometric lens with base radius of curvature of $\sim 8 \mu \mathrm{m}$ located centrally over the fiber core. This has the effect of producing a beam waist that is $\sim 10-15 \mu \mathrm{m}$ in front of the fiber end face and with a diameter that is less than the fiber core itself, as shown in Fig. 3. If the fiber is aligned to the component such that this waist is positioned to overlap the device end-face facet, best coupling to and from the device is achieved. There is a tradeoff with this design since the fiber mode and coupling device beam mode may not be of the same $\mathrm{M}^{\wedge} 2$ value. With differing $\mathrm{M}^{\wedge} 2$ values, the fiber lens geometry is chosen to either match the beam waist diameters, or the far-field numeric apertures.

NRE costs for custom package designs can be prohibitive. For this reason, the final package design was based around a simple machined Gold-over-Nickel Kovar bath tub package, with associated fiber feed-through pipes and wire-bond pin headers being attached to the package via a hot epoxy wick technique. Hot-wicking at $150{ }^{\circ} \mathrm{C}$ ensures that the epoxy glass transition temperature $T_{G}$ is above the reflow temperature of the 


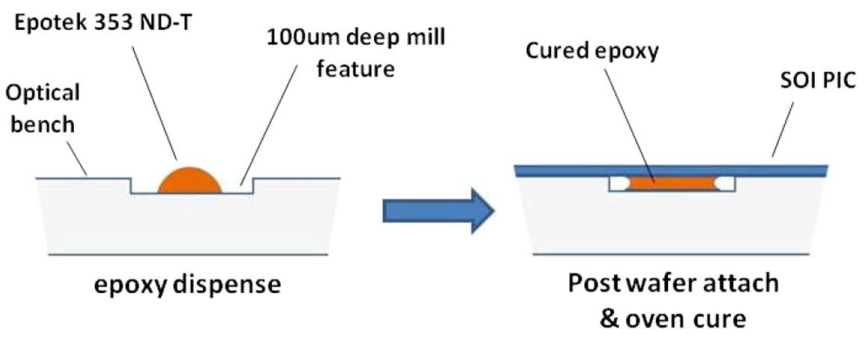

Fig. 4. Schematic of epoxy dispense for SOI PIC attach.

solders that would be used for TEC and fiber tube attach. After package assembly, solder attach of the TEC and optical bench was carried out using Bismuth/Tin (58Bi42Sn) solder having a reflow temperature of $138^{\circ} \mathrm{C}$. Specially designed high-temperature plastic formers were used inside each package to ensure that both the optical bench and TECs were held accurately in place during solder reflow. Due to the dimensions of the optical bench, three TEC units were soldered in the package and electrically connected in parallel to provide sufficient device coverage.

With the absence of wafer back-side metallization, and the need to keep wafer temperature exposure below $\sim 70{ }^{\circ} \mathrm{C}$, thermal cure epoxies were investigated for their suitability for photonic integrated circuit (PIC) attach to the planar optical bench. For thermally conducting epoxies/adhesives to function optimally, a high-temperature oven cure step is required $\left(150-200^{\circ} \mathrm{C}\right)$. This ensures that the conducting filler in the adhesive will efficiently cross link for good thermal performance. If the cure temperature is too low, the insulating resin in the adhesive will inhibit good cross linking, resulting in poor thermal performance after cure. For epoxy dispense, the traditional method for large area coverage is to use a screen printing technique to produce a thin uniform epoxy layer, prior to device placement. This could readily be carried out with the optical bench outside the package, but with this having previously been soldered attached inside the package, this option was not available.

Two differing bonding methods were evaluated. The first AOWC module was assembled using a uniform layer of thermally conducting Epotek H20-e epoxy. This epoxy is quite granular so to enhance even spreading. The optical bench and module were placed on a hot plate at $60^{\circ} \mathrm{C}$ to reduce the epoxy viscosity and promote even flow. The waveguide was then carefully placed on top of this. The whole assembly was oven cured overnight at $60{ }^{\circ} \mathrm{C}$. The second method employed the same planar optical bench, but with the addition of an array of $100 \mu \mathrm{m}$ deep mill holes, into which a controlled amount of viscous adhesive was deposited. This is illustrated in Fig. 4. The waveguide was placed on the planar surface and so brought into contact with six adhesive spots. Once cured, the adhesive shrinkage would provide a mild compressive force to keep the waveguide substrate in physical contact with the bench with no adhesive interface layer being present outside of the mill areas. In this way, there is a completely planar contact area between the optical bench and then SOI device and also physical contact between the tile area directly under the SOA and the optical bench for thermal transfer during operation.

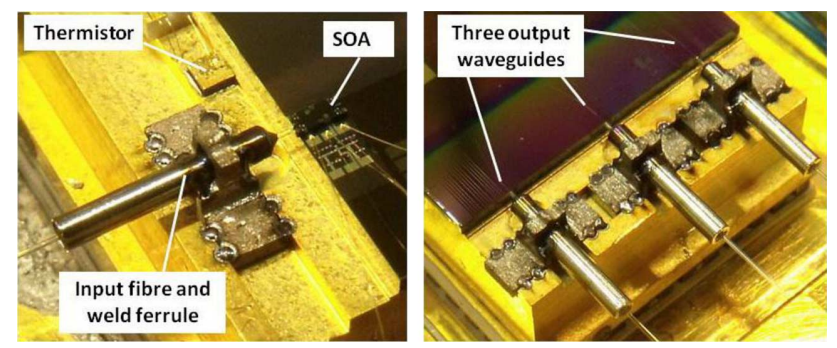

Fig. 5. Aligned and welded input and output fibers.
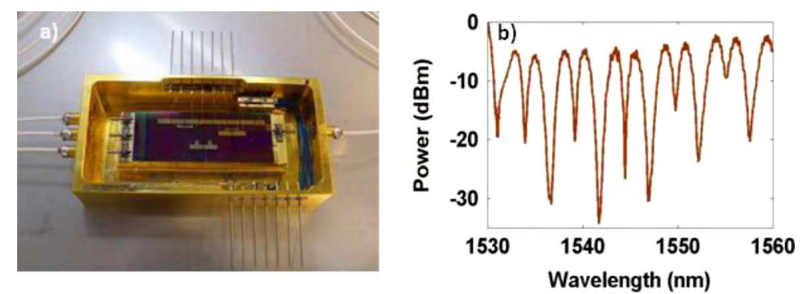

Fig. 6. (a) Packaged and pigtailed hybrid AOWC. (b) DI spectral responses.

Comparing the thermal expansion of the optical bench material (Kovar, $5.9 \mathrm{ppm} /{ }^{\circ} \mathrm{C}$ ), with the PIC attach epoxy (Epotek $353 \mathrm{ND}-\mathrm{T}, 43 \mathrm{ppm} /{ }^{\circ} \mathrm{C}$ ), there is a mild compressive force between bench and device wafer, after cool-down from the oven cure process, ensuring that physical contact is maintained during device operation. Epoxy dispense was regulated via an EFD pneumatic dispense unit. This allows very small and repeatable epoxy volumes to be dispensed locally in the center of each mill feature.

Fiber align was carried out using standard submicrometer four-axis alignment system, which also incorporates a Lumonics pulsed YAG laser system for fiber fixing. Each SOI module was loaded onto the package holder stage on the alignment workstation, with sufficient electrical current supplied to generate spontaneous emission from the SOA. This emission would be available at the input of the SOA to allow alignment of the input fiber, and also available at each of the three output waveguide end faces for alignment of each of the output fibers. Fig. 5 shows the aligned and welded fibers. The operation of the thermistor was checked by placing a small thermocouple on the SOI wafer surface near to the SOA and comparing it to the reading from the on-board thermistor. We determined that the initial placement of the device thermistor introduced a temperature offset which increased at higher SOA drive currents and that at a constant $180 \mathrm{~mA}$ drive current, the fiber aligned optical power was not constant, but reduced over time. This observation let to the conclusion that there was local heating of the SOA due to insufficient thermal transfer away from the SOA through to the optical bench. With this observation, the rest hybrid AOWC modules were assembled using the mill-array optical bench with discrete epoxy dispense, instead of H20-e epoxy, as shown in Fig. 4. The thermocouple was soldered to the optical bench prior to subsequent assembly steps and when the fibers were aligned, the modules did not exhibit any power drop over time, leading to the conclusion that this method of PIC attach produced superior thermal performance. Fig. 6(a) shows the packaged and pigtailed hybrid AOWC, while Fig. 6(b) shows the DI spectral responses after performing wavelength tuning. 


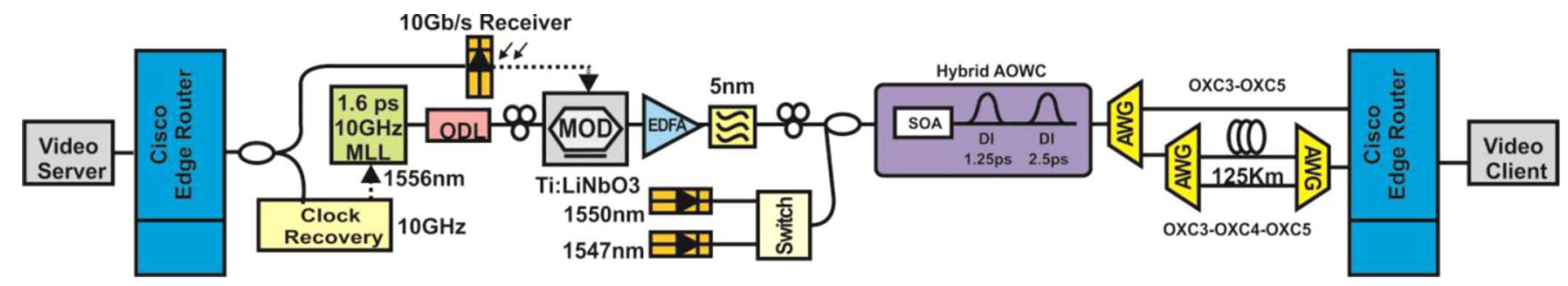

Fig. 7. Experimental setup for video streaming routing.
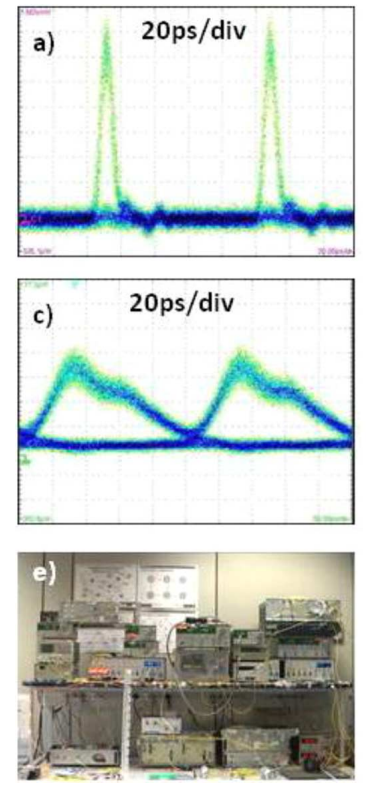

Fig. 8. (a) Video RZ back-to-back (B2B) signal. (b) Wavelength converted signal after OXC3-OXC5 at $\lambda_{1}=1547$. (c) Wavelength converted signal at $\lambda_{2}=1550$ after OXC2-OXC4-OXC5. (d) Spectra from wavelength converted signals. (e) Network setup overview. (f) Video performance over the WDM link.

\section{EXPERIMENT}

\section{A. $10 \mathrm{~Gb} / \mathrm{s}$ Path Video Streaming Routing}

Fig. 7 illustrates the experimental setup for the video streaming routing. It is comprised of four main blocks: the video server, the video client, the edge routing interface, and the optical switching network. The video traffic provided by the edge IP router optical interface is divided first with a $3 \mathrm{~dB}$ coupler. Two paths follow next. The first is used for clock recovery and triggering a $10 \mathrm{GHz}$ mode-locked laser (MLL) at $1556 \mathrm{~nm}$, while the second one is employed for real traffic opto-electronic conversion using a $10 \mathrm{~Gb} / \mathrm{s}$ optical receiver. The generated electrical signal modulates the $1.6 \mathrm{ps}$ optical pulses provided by the MLL using a LiNbO3 modulator. The data sequence is combined then with the output of an optical switch driven by two laser diode pumps at 1547 and $1550 \mathrm{~nm}$, respectively, before launched into the hybrid AOWC. The data sequence (pump signal) interacts into the SOA with the laser diode (probe signal) resulting in an inverted wavelength conversion via cross-gain modulation (XGM). The cross-phase modulation (XPM) produces subsequently a chirped wavelength converted signal with red-shifted leading edges and blue-shifted trailing edges. The first DI element of the hybrid

\begin{tabular}{|l|r|}
\hline Name & Unitl:3:1 (MU120118A) Accumulated \\
\hline Transmitted Bit Rate (bit/s) & $6,258,144,791 \mathrm{bit} / \mathrm{s}$ \\
\hline Transmitted Bit Rate (\%) & $62.58 \%$ \\
\hline Transmitted Rate (\%) & $82.14 \%$ \\
\hline Transmitted Byte & $70,404,128,896$ \\
\hline Transmitted Frame & $1,100,064,514$ \\
\hline Transmitted Frame (fps) & $12,222,939 \mathrm{fps}$ \\
\hline Transmitted IPv4 Packet & 0 \\
\hline Transmitted IPv4 Packet (pps) & $0 \mathrm{pps}$ \\
\hline Transmitted ARP Reply & 0 \\
\hline Transmitted ARP Request & 0 \\
\hline Transmitted Ping Reply & 0 \\
\hline Transmitted Ping Request & 0 \\
\hline Received Bit Rate (bit/s) & $6,258,144,786 \mathrm{bit} / \mathrm{s}$ \\
\hline Received Bit Rate (\%) & $62.58 \%$ \\
\hline Received Rate (\%) & $82.14 \%$ \\
\hline Received Byte & $70,404,128,846$ \\
\hline Received Frame & $1,100,064,505$ \\
\hline Received Frame (fps) & $12,222,939 \mathrm{fps}$ \\
\hline
\end{tabular}

Fig. 9. Packet loss QoS analysis.

AOWC is employed for selecting the blue-shifted sideband of the converted signal accelerating significantly its recovery time. The second DI is operated then as a notch filter for carrier frequency suppression and signal polarity inversion. Depending on the pump wavelength used into the AOWC, video traffic is routed either directly to the end IP router through OXC3-OXC5 $(\lambda 1=1547 \mathrm{~nm})$ or through a dispersion compensated DWDM link with $125 \mathrm{~km} \mathrm{G.652} \mathrm{fiber} \mathrm{spools}(\lambda 2=1550 \mathrm{~nm})$ following the route OXC2-OXC4-OXC5. In both cases, the SOA is driven with $140 \mathrm{~mA}$, temperature is maintained at 20 ${ }^{\circ} \mathrm{C}$, while the detuning of the DIs with respect to the probe wavelength carrier is $\sim 0.2 \mathrm{~nm}$. The average optical power for the probe signal is measured $\sim 8 \mathrm{dBm}$, while for the $10 \mathrm{~Gb} / \mathrm{s}$ pump signal $\sim 6 \mathrm{dBm}$. The transmitted and switched video streaming sequence is subsequently evaluated with service quality measurements.

Fig. 8(a) shows the incoming $10 \mathrm{G}$ return-to-zero (RZ) video data stream injected into the SOA for XGM-XPM. Fig. 8(b) illustrates the wavelength converted signal at $\lambda 1=1547 \mathrm{~nm}$ passing through $\mathrm{OXC} 3-\mathrm{OXC} 5$ when properly detuning the AOWC DI filter characteristics for effective chirp filtering and signal polarity inversion. The pulse broadening observed is mainly attributed to the narrow AWG channel filtering $(0.3 \mathrm{~nm})$. Fig. 8(c) depicts the wavelength converted signal at $\lambda_{2}=1550$ $\mathrm{nm}$ passing through $\mathrm{OXC2}-\mathrm{OXC} 4-\mathrm{OXC} 5$ encompassing a DWDM link. A clear and open eye has been recorded but with large broadening due to the cascading filtering and incomplete dispersion compensation in the DWDM configuration. Fig. 8(d) 


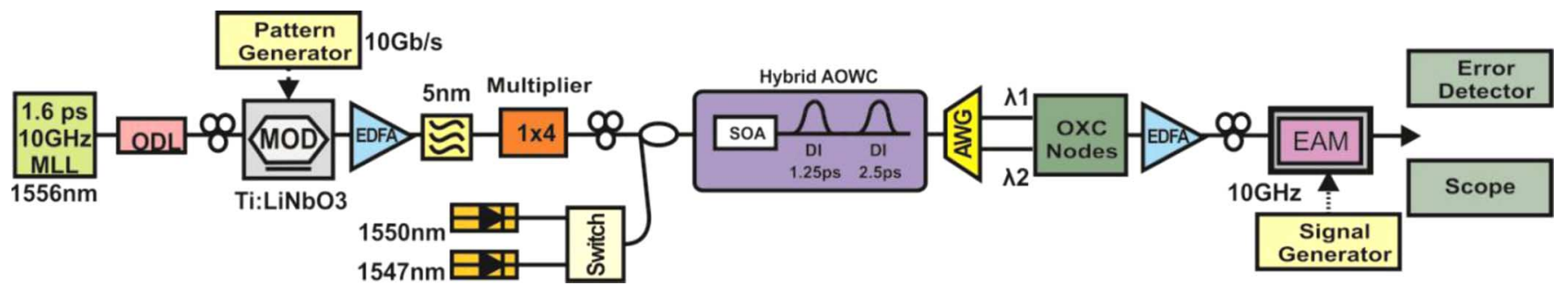

Fig. 10. Experimental setup for $40 \mathrm{~Gb} / \mathrm{s}$ data switching.

depicts the spectra obtained from the two wavelength converted signals, while Fig. 8(e) illustrates the optical network setup comprising the hybrid integrated AOWC, the DFB laser bank, the optical wavelength switch, and intermediate amplification stages for power compensation. Fig. 8(f) shows a typical video frame of the data stream routed over the $125 \mathrm{~km}$ DWDM link where no evidence of packet loss and signal degradation was observed. Fig. 9 depicts the QoS verification with packet loss end-to-end measurement analysis on the IP router packet optical interface. Only nine frames were counted lost over the whole measurement period but with no significant impact on QoS performance. The video streaming process was realized during switching operation and some seconds of video freezing image were encountered. Ethernet protocol stops counters when the link is down and multicast protocol resynchronization is performed. The switching time between the two wavelength states was less than $5 \mathrm{~s}$, video streaming was automatically recovered, and during operation there was no need to tune filters or optimize the AOWC.

\section{B. $40 \mathrm{~Gb} / \mathrm{s}$ Path Data Switching}

For testing the switching capabilities of the hybrid AOWC at $40 \mathrm{~Gb} / \mathrm{s}$, the experimental setup had to be modified as shown in Fig. 10, since $40 \mathrm{~Gb} / \mathrm{s}$ optical interfaces were not available for IP edge router interconnecting. In the transmitter, $1.6 \mathrm{ps}$ optical pulses were generated by an MLL at $1556 \mathrm{~nm}$ and encoded with a $2^{7}-1$ pseudorandom binary sequence (PRBS) using a LiNbO3 modulator. Then, the optical signal was time-multiplexed through a fiber interleaver in order to constitute a $40 \mathrm{~Gb} / \mathrm{s}$ data stream (pump signal). The generated data sequence was combined with the output of the laser diode pump stack emitting at wavelengths 1547 and $1550 \mathrm{~nm}$, respectively. The flip-chip mounted SOA of the AOWC was used for the nonlinear processing by inducing chirp in the converted probe signal due to the refractive index modulation caused by the pulsed pump signal. The first SOI Mach-Zehnder interferometer (MZI) filter was detuned with respect to the probe wavelength and by filtering the blue (fast) chirp the acceleration of the effective recovery time was achieved. The second SOI-MZI filter was employed to restore the polarity of the optical pulses by suppressing the carrier of the probe signal. Depending on the pump wavelength used into the AOWC, data bit stream was routed either through OXC3-OXC5 at wavelength $\lambda_{1}=1547 \mathrm{~nm}$ or through OXC2-OXC4-OXC6-OXC5 at $\lambda_{2}=1550 \mathrm{~nm}$. The wavelength converted and routed signals were evaluated with BER measurements after performing optical demultiplexing using an electro-absorption modulator.
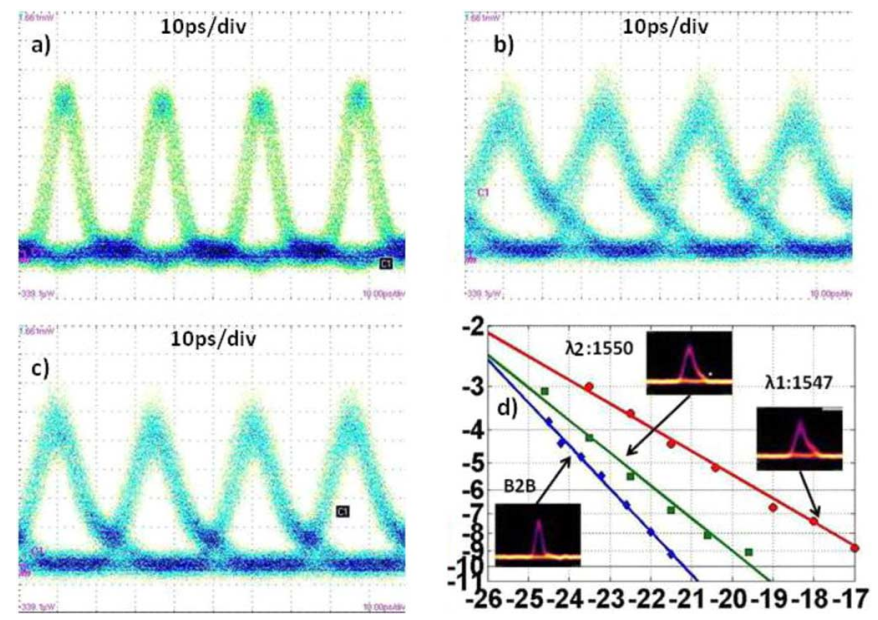

Fig. 11. Eye diagrams of (a) $40 \mathrm{~Gb} / \mathrm{s}$ (B2B) signal. (b) Wavelength converted signal after OXC3-OXC5 at $\lambda_{1}=1547$. (c) Wavelength converted after OXC2-OXC4-OXC6-OXC5 signal at $\lambda_{2}=1550$. (d) BER measurements $[\log (\mathrm{BER})-\mathrm{P}(\mathrm{dBm})]$

Fig. 11(a) shows the incoming $40 \mathrm{~Gb} / \mathrm{s} \mathrm{RZ}$ data signal injected into the SOA for XGM-XPM. Fig. 11(b) illustrates the wavelength converted signal at $\lambda_{1}=1547 \mathrm{~nm}$ passing through $\mathrm{OXC} 3-\mathrm{OXC} 5$. The pulse broadening is mainly attributed to the narrow AWG channel filtering while the pulse asymmetry observed to the AWG spectral mismatch with the DI filter comb. Fig. 11(c) depicts the wavelength converted signal at $\lambda_{2}=1550 \mathrm{~nm}$ passing through the OXC3-OXC4-OXC6-OXC5. A clear and open eye has been recorded with better performance from $\lambda_{1}$ at 1547 due to the symmetrical filtering from both AWG and DI responses as well as to the better amplification properties. Fig. 11(d) depicts the BER measurements obtained for both wavelength converted and routed signals. Less than $5 \mathrm{~dB}$ penalty has been measured for the path $\lambda_{1}=1547 \mathrm{~nm}$ with no evidence of error floor until 10-9. The error floor was mainly attributed to the narrow AWG filtering resulting to pulse overlapping as well as to the mismatch of AWG and DI spectral responses. Error-free performance with less than $2 \mathrm{~dB}$ power penalty was achieved for the path $\lambda_{2}=1550 \mathrm{~nm}$.

Dynamic wavelength changes have been implemented as well at $40 \mathrm{~Gb} / \mathrm{s}$. The AOWC was optimized first for both transmission wavelength states and then dynamic switching was performed. The switching time was around $5 \mathrm{~s}$ and during operation time there was no need to tune filters or make any AOWC corrections. During sequential wavelength changes, BER performance was deteriorating due to polarization changes that were limiting the operational performance of our integrated AOWC. 


\section{CONCLUSION}

We have system tested a packaged and pigtailed hybrid SOI AOWC on a meshed network testbed. The photonic chip performs wavelength conversion using a flip-chip mounted SOA and two monolithically integrated DIs. The device has been inserted in a GMPLS network emulating five nodes interconnected by bundles of bidirectional fibers. We have demonstrated $10 \mathrm{GbE}$ path video-streaming routing and 40 $\mathrm{Gb} / \mathrm{s}$ data switching using different pump signals to assist the wavelength conversion process. Error-free transmission and different kind of end-to-end services (video distribution, audio conference, and video conference) have been successfully evaluated.

\section{ACKNOWLEDGMENT}

The authors would like to thank A. Labs for providing supporting equipment.

\section{REFERENCES}

[1] N. Leavitt, "Network-usage changes push internet traffic to the edge," Computer, vol. 43, no. 10, pp. 13-15, Oct. 2010.

[2] J. Gripp, J. E. Simsarian, J. D. LeGrange, P. G. Bernasconi, and D. T. Neilson, "Architectures, components, and subsystems for future optical packet switches," IEEE J. Sel. Topics Quantum Electron., vol. 16, no. 5, pp. 1394-1404, Sep./Oct. 2010.

[3] N. Beheshti, E. Burmeister, Y. Ganjali, J. E. Bowers, D. J. Blumenthal, and N. McKeown, "Optical packet buffers for backbone internet routers," IEEE/ACM Trans. Netw., vol. 18, no. 5, pp. 1599-1609, Oct. 2010.

[4] E. Bonetto, L. Chiaraviglio, G. A. Castillo, D. Cuda, and F. Neri, "Optical technologies can improve the energy efficiency of networks," in Proc. 35th Eur. Conf. Opt. Commun., Vienna, Austria, Sep. 2009, pp. $1-4$.

[5] R. S. Tucker, R. Parthiban, J. Baliga, K. Hinton, R. W. A. Ayre, and W. V. Sorin, "Evolution of WDM optical IP networks: A cost and energy perspective," J. Lightw. Technol., vol. 27, no. 3, pp. 243-252, Feb. 2009.

[6] S. J. Ben Yoo, "Energy efficiency in the future internet: The role of optical packet switching and optical-label switching," IEEE J. Sel. Topics Quantum Electron., vol. 17, no. 2, pp. 406-418, Mar./Apr. 2011.

[7] N. M. M. K. Chowdhury and R. Boutaba, "Network virtualization: State of the art and research challenges," IEEE Commun. Mag., vol. 47, no. 7, pp. 20-26, Jul. 2009.
[8] D. J. Blumenthal, J. Barton, N. Beheshti, J. E. Bowers, E. Burmeister, L. A. Coldren, M. Dummer, G. Epps, A. Fang, Y. Ganjali, J. Garcia, B. Koch, V. Lal, E. Lively, J. Mack, M. Masanovic, N. McKeown, K. Nguyen, S. C. Nicholes, H. Park, B. Stamenic, A. Tauke-Pedretti, H. Poulsen, and M. Sysak, "Integrated photonics for low-power networking," IEEE J. Sel. Topics Quantum Electron., vol. 17, no. 2, pp. 458-471, Jan. 2011.

[9] R. Nagarajan, M. Kato, J. Pleumeekers, P. Evans, S. Corzine, S. Hurtt, A. Dentai, S. Murthy, M. Missey, R. Muthiah, R. A. Salvatore, C. Joyner, R. Schneider, M. Ziari, F. Kish, and D. Welch, "InP photonic integrated circuits," IEEE J. Sel. Topics Quantum Electron., vol. 16, no. 5, pp. 1113-1125, Oct. 2010.

[10] Y. Vlasov, W. M. J. Green, and F. Xia, "High-throughput silicon nanophotonic wavelength-insensitive switch for on-chip optical networks," Nature Photon., vol. 2, pp. 242-246, Mar. 2008.

[11] D. Liang, A. W. Fang, H.-W. Chen, M. Sysak, B. R. Koch, E. Lively, O. Raday, Y.-H. Kuo, R. Jones, and J. E. Bowers, "Hybrid silicon evanescent approach to optical interconnects," Appl. Phys. A, Mater. Sci. Process., vol. 95, no. 4, pp. 1045-1057, Feb. 2009, 2009.

[12] L. Liu, T. Spuesens, G. Roelkens, D. Van Thourhout, P. Regreny, and P. Rojo-Romeo, "A thermally tunable microdisk laser built on a III-V/silicon-on-insulator heterogeneous integration platform," IEEE Photon. Technol. Lett., vol. 22, no. 17, pp. 1270-1272, Sep. 2010

[13] T. Aalto, M. Harjanne, M. Kapulainen, S. Ylinen, J. Ollila, V. Vilokkinen, L. Moerl, M. Moehrle, and R. Hamelin, "Integration of InP-based optoelectronics with silicon waveguides," in Proc. SPIE, San Jose, CA, 2009, vol. 7218, pp. 72180O-1-72180O-14.

[14] L. Zimmermann, G. B. Preve, K. Voigt, G. Winzer, J. Kreissl, L. Moerl, C. Stamatiadis, L. Stampoulidis, and H. Avramopoulos, "High-precision flip-chip technology for all-optical wavelength conversion using SOI photonic circuit," in Proc. 8th IEEE Int. Conf. Group IV Photon., London, U.K., 2011, pp. 237-239.

[15] C. Stamatiadis, L. Stampoulidis, D. Kalavrouziotis, I. Lazarou, K. Vyrsokinos, L. Zimmermann, K. Voigt, G. B. Preve, L. Moerl, J. Kreissl, and H. Avramopoulos, "Fabrication and experimental demonstration of the first $160 \mathrm{~Gb} / \mathrm{s}$ hybrid silicon-on-insulator integrated all-optical wavelength converter," Opt. Exp., vol. 20, pp. 3825-3831, Jan. 2012.

[16] E. Ciaramella, "Achievements and future prospects of wavelength conversion and all-optical regeneration," presented at the presented at the Opt. Fiber Commun. Conf., Los Angeles, CA, 2011, Paper OMT1.

[17] F. Gomez-Agis, O. Raz, S. J. Zhang, E. Tangdiongga, L. Zimmermann, K. Voigt, C. Vyrsokinos, L. Stampoulidis, and H. J. S. Dorren, "All optical wavelength conversion at $160 \mathrm{Gbit} / \mathrm{s}$ using SOA and silicon-on-insulator photonic circuit," Electron. Lett., vol. 45, no. 22, pp. 1132-1133, Oct. 2009.

Author biographies not included at author request due to space constraints. 